\title{
An identity conjectured by Lacasse via the tree function
}

\author{
Helmut Prodinger* \\ Department of Mathematics \\ University of Stellenbosch \\ 7602 Stellenbosch, South Africa \\ hproding@sun.ac.za
}

Submitted: April 18, 2013; Accepted: July 18, 2013; Published: July 26, 2013

Mathematics Subject Classifications: 05A15, 05A19.

\begin{abstract}
A. Lacasse conjectured a combinatorial identity in his study of learning theory. Various people found independent proofs. Here is another one that is based on the study of the tree function, with links to Lamberts $W$-function and Ramanujan's $Q$-function. It is particularly short.
\end{abstract}

Let

$$
\begin{aligned}
\xi(n) & =\sum_{k=0}^{n}\left(\begin{array}{l}
n \\
k
\end{array}\right)\left(\frac{k}{n}\right)^{k}\left(\frac{n-k}{n}\right)^{n-k}, \\
\xi_{2}(n) & =\sum_{k_{1}+k_{2}+k_{3}=n} \frac{n !}{k_{1} ! k_{2} ! k_{3} !}\left(\frac{k_{1}}{n}\right)^{k_{1}}\left(\frac{k_{2}}{n}\right)^{k_{2}}\left(\frac{k_{3}}{n}\right)^{k_{3}} .
\end{aligned}
$$

Then Lacasse [3] conjectured that $\xi_{2}(n)=\xi(n)+n$. There are now three independent proofs [6, 1, 5]. Here, we want to shed additional light on the matter, by using the tree function (equivalent to Lambert's $W$-function [7]) and linking the enumeration to the celebrated $Q$-function of Ramanujan [2].

As Younsi has already pointed out, it is easier to work with

$$
\begin{aligned}
& \alpha(n)=n^{n} \xi(n)=\sum_{k=0}^{n}\left(\begin{array}{l}
n \\
k
\end{array}\right) k^{k}(n-k)^{n-k}, \\
& \beta(n)=n^{n} \xi_{2}(n)=\sum_{k_{1}+k_{2}+k_{3}=n} \frac{n !}{k_{1} ! k_{2} ! k_{3} !} k_{1}^{k_{1}} k_{2}^{k_{2}} k_{3}^{k_{3}} .
\end{aligned}
$$

${ }^{*}$ The author was supported by an incentive grant of the NRF of South Africa. 
The tree function [4] $y(z)$ is defined by $y=z e^{y}$ and possesses the expansion

$$
y(z)=\sum_{n \geqslant 1} n^{n-1} \frac{z^{n}}{n !} .
$$

Therefore

$$
\sum_{n \geqslant 0} n^{n} \frac{z^{n}}{n !}=1+z y^{\prime}(z)=1+\frac{y}{1-y}=\frac{1}{1-y} .
$$

Consequently,

$$
\alpha(n)=n !\left[z^{n}\right]\left(\frac{1}{1-y}\right)^{2} \text { and } \beta(n)=n !\left[z^{n}\right]\left(\frac{1}{1-y}\right)^{3} .
$$

Now we compute the coefficients of a general power via Cauchy's integral formula:

$$
\begin{aligned}
{\left[z^{n}\right]\left(\frac{1}{1-y}\right)^{d} } & =\frac{1}{2 \pi i} \oint \frac{d z}{z^{n+1}}\left(\frac{1}{1-y}\right)^{d} \\
& =\frac{1}{2 \pi i} \oint \frac{d y(1-y) e^{-y} e^{y(n+1)}}{y^{n+1}}\left(\frac{1}{1-y}\right)^{d} \\
& =\left[y^{n}\right] \frac{e^{n y}}{(1-y)^{d-1}} \\
& =\sum_{k=0}^{n} \frac{n^{n-k}}{(n-k) !}\left(\begin{array}{c}
k+d-2 \\
d-2
\end{array}\right) .
\end{aligned}
$$

Therefore

$$
\alpha(n)=\sum_{k=0}^{n} \frac{n !}{k !} n^{k}, \quad \beta(n)=\sum_{k=0}^{n} \frac{n !}{k !}(n+1-k) n^{k},
$$

and

$$
\begin{aligned}
\beta(n)-\alpha(n) & =\sum_{k=0}^{n} \frac{n !}{k !}(n-k) n^{k} \\
& =\sum_{k=0}^{n} \frac{n !}{k !} n^{k+1}-\sum_{k=1}^{n} \frac{n !}{(k-1) !} n^{k}=n^{n+1},
\end{aligned}
$$

as claimed.

Note that $\alpha(n)$ is linked to Ramanujan's celebrated $Q$-function [2] via

$$
\alpha(n)=n^{n}(1+Q(n)) .
$$




\section{References}

[1] W. Y. C. Chen, J. F. F. Peng and H. R. L. Yang, Decomposition of triply rooted trees, Electronic Journal of Combinatorics 20, Issue 2 (2013), P10.

[2] P. Flajolet, P. Grabner, P. Kirschenhofer and H. Prodinger, On Ramanujan's $Q(n)-$ function, Journal of Computational and Applied Mathematics, 58:103-116, 1995.

[3] A. Lacasse, Bornes PAC-Bayes et algorithmes d'apprentissage, Ph. D. thesis, Université Laval, Quebec, 2010.

[4] I. Goulden and D. Jackson, Combinatorial Enumeration, Wiley, 1983.

[5] Y. Sun, A simple proof of an identity conjectured by Lacasse, Electronic Journal of Combinatorics 20, Issue 2 (2013), P11.

[6] M. Younsi, Proof of a combinatorial conjecture coming from the PAC-Bayesian machine learning theory, arXiv:1209.0824.

[7] R. Corless, G. Gonnet, D. Hare, D. Jeffrey and D. Knuth, On the Lambert Wfunction, Advances in Computational Mathematics 5: 329-359, 1996. 